\title{
Opfer zweiter Klasse - gutta cavat lapidem
}

Opferangehörige in Staatshaftungsfall erstmals zur Beschwerde gegen Verfahrenseinstellung zugelassen

Marc Thommen *

Anmerkung zum Beschluss des Obergerichts des Kantons Zürich, III. Strafkammer, UE170255 vom 24. April 2018 (Beschwerde gegen die Verfügung der Staatsanwaltschaft): Im Gefolge einer Strafuntersuchung gegen das Ärzte- und Pflegepersonal des Universitätsspitals Zürich wegen mutmasslich fataler Behandlungsfehler lässt das Obergericht des Kantons Zürich eine Opferangehörige erstmals zur Beschwerde gegen eine Verfahrenseinstellung zu. Dies ist deshalb bemerkenswert, weil das Bundesgericht in ständiger Rechtsprechung davon ausgeht, dass Opfer, die von staatlichen Akteuren geschädigt wurden, keine zivilen, sondern bloss öffentlichrechtliche Haftungsansprüche geltend machen können. Sie sollen deshalb nicht berechtigt sein, sich gegen Verfahrenseinstellungen zu wehren.

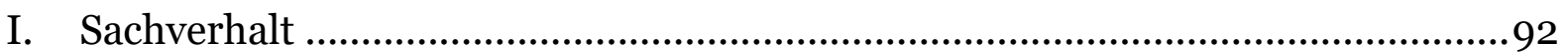

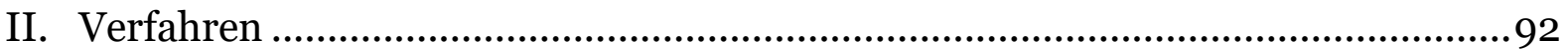

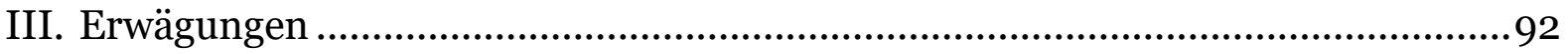

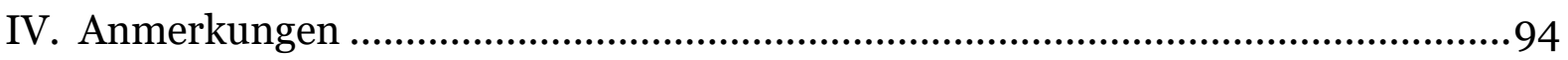

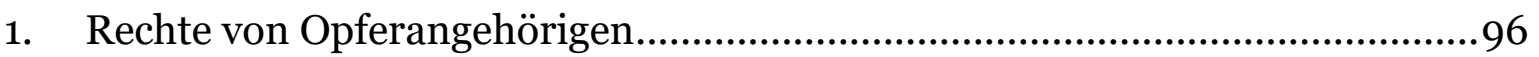

2. Konstituierung als Privatklägerin ................................................................96

3. Interpretation der Zivilansprüche ……………..............................................97

4. Legitimation zur Beschwerde....................................................................100

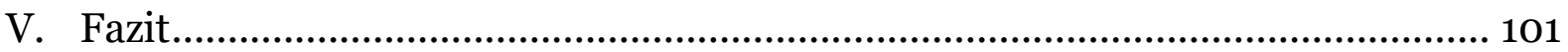

Zitiervorschlag: Marc Thommen, Opfer zweiter Klasse - gutta cavat lapidem, in: suigeneris 2019, S. 91

URL: $\quad$ sui-generis.ch/95

DOI: $\quad$ https://doi.org/10.21257/sg.95


ber für Strafrecht und Strafprozessrecht unter Einschluss des Wirtschafts- und Verwaltungsstrafrechts an der Universität Zürich. Herzlich zu danken habe ich RA Martina Jaussi, MLaw sowie RA Dr. iur. Christina Galeazzi für ihre luziden Inputs zum Manuskript. Verbleibende Unzulänglichkeiten gehen alleine auf mich.

Dieses Werk ist lizenziert unter einer Creative Commons Namensnennung - Weitergabe unter gleichen Bedingungen 4.0 International Lizenz. 


\section{Sachverhalt}

1 Am 5. August 2015 wurde ein 78-jähriger Mann im Universitätsspital Zürich (USZ) wegen Leberkrebs operiert. In den frühen Morgenstunden des 20. August 2015 erlitt er einen Herzstillstand und als Folge davon einen schweren Hirnschaden. Noch vor Ende August 2015 verstarb er im USZ.

\section{Verfahren}

2 Die Tochter des Verstorbenen erstattete am 27. November 2015 Strafanzeige gegen das behandelnde Ärzte- und Pflegepersonal des USZ wegen fahrlässiger schwerer Körperverletzung und fahrlässiger Tötung. Die Staatsanwaltschaft Zürich eröffnete am 2. Februar 2016 eine Strafuntersuchung gegen Unbekannt. In der Folge holte sie ein rechtsmedizinisches Gutachten ein. Mit Verfügung vom 28. August 2017 stellte die Staatsanwaltschaft das Verfahren ein. Aufgrund des Gutachtens hätten keine Hinweise auf eine Sorgfaltspflichtverletzung des Spitalpersonals und folglich kein Verdacht auf ein strafbares Verhalten vorgelegen. Gegen diese Einstellung erhob die Tochter am 13. September 2017 Beschwerde.

Das Obergericht trat - mit nachfolgend $\mathrm{zu}$ besprechender Begründung (III.) auf die Beschwerde ein. In der Sache teilte das Obergericht die Einschätzung der Staatsanwaltschaft, wonach aus rechtsmedizinischer Sicht keine Hinweise auf Sorgfaltswidrigkeiten des Spitalpersonals vorlagen und wies die Beschwerde ab.

\section{Erwägungen}

4 1.1. Der Verstorbene ist Opfer im Sinn von Art. 116 Abs. 1 StPO 1 [...] Die Beschwerdeführerin ist Tochter des Verstorbenen und gehört als solche zum Kreis der nahen Angehörigen des Opfers im Sinn von Art. 116 Abs. 2 StPO.

$5 \quad 1.2$ Den Opferangehörigen stehen die gleichen Rechte wie dem Opfer zu, sofern sie Zivilansprüche geltend machen (Art. 117 Abs. 3 StPO) [...]. Die Opferangehörigen müssen eigene Zivilansprüche gegen den Straftäter geltend machen (Art. 122 Abs. 2 StPO). In Betracht kommen in erster Linie Versorgerschaden (Art. 45 Abs. 3 OR ${ }^{2}$ ) und Genugtuung (Art. 47 OR).

6 Im vorliegenden Fall steht eine mutmasslich begangene Straftat im Rahmen einer medizinischen Behandlung am Universitätsspital Zürich (USZ) zur Diskussion. Beim USZ handelt es sich um eine Anstalt des kantonalen öffentlichen Rechts mit eigener Rechtspersönlichkeit (§ 1 USZG3). Im Kanton Zürich kommt bei Haftungsansprüchen gegenüber Organisationen des kantonalen öffentlichen Rechts mit eigener Rechtspersönlichkeit bzw. gegenüber ihren Organen und gegenüber den in ihrem Dienst stehenden Personen, soweit sie öffentlich-rechtliche Verrichtungen ausüben, das Gesetz vom 14. September 1969 über die Haftung des Staates und der Gemeinden sowie ihrer Behörden und Beamten zur Anwendung

$1 \overline{\text { Schweizerische Strafprozessordnung vom 5. Okt- }}$ ober 2007 (StPO; SR 312.0)

2 Bundesgesetz betreffend die Ergänzung des Schweizerischen Zivilgesetzbuches (Fünfter Teil: Obligationenrecht) vom 30. März 1911 (OR; SR 220)

3 Gesetz über das Universitätsspital Zürich vom 19. September 2005 (USZG; LS 813.15). 
(§ 3 Haftungsgesetz 4 ). Der geschädigten Person steht kein Anspruch gegen die Angestellten zu (§4 i.V.m. § 6 Abs. 4 Haftungsgesetz). Demnach sind allfällige Forderungen der Beschwerdeführerin nicht zivilrechtlicher, sondern öffentlichrechtlicher Natur. [...]

7 Dies führt zur Frage, ob den Opferangehörigen, die anstelle einer zivilrechtlichen eine öffentlich-rechtliche Forderung geltend machen, aufgrund des Wortlauts von Art. 117 Abs. 3 StPO die Berechtigung zur Anfechtung einer Nichtanhandnahme- oder Einstellungsverfügung fehlt [...].

$8 \quad 1.3$ Die Voraussetzung der Geltendmachung von Zivilansprüchen wird auch zur Eingrenzung der Legitimation der Privatklägerschaft zur Beschwerde in Strafsachen ans Bundesgericht (Art. $78 \mathrm{ff}$. BGG5) verwendet. [...] Zu den Beschwerdeberechtigten gehört u.a. die Privatklägerschaft, wobei für sie zusätzlich vorausgesetzt ist, dass sich der angefochtene Entscheid auf die Beurteilung ihrer Zivilansprüche auswirken kann (Art. 81 Abs. 1 lit. b Ziff. 5 BGG). Macht die geschädigte Person anstelle von Zivilansprüchen öffentlich-rechtliche Forderungen aus Staatshaftung geltend, tritt das Bundesgericht, von Ausnahmen bei Gewaltdelikten abgesehen [...], auf die Beschwerde nicht ein (BGer, Urteil 6B_1074/2017 vom 27.11.17 E. 2 [...]).

9 In der Lehre wird diese restriktive Legitimationspraxis kritisiert. Dagegen wird hauptsächlich vorgebracht, der Entscheid

Haftungsgesetz des Kantons Zürich vom 14. September 1969 (LS 170.1).

5 Bundesgesetz über das Bundesgericht vom 17. Juni 2005 (BGG; SR 173.110). im Strafpunkt könne nicht nur auf Zivilforderungen, sondern auch auf öffentlich-rechtliche Haftungsansprüche der geschädigten Person gegen den Staat Auswirkungen haben [...].

10 1.4 Diese Kritik lässt sich auf Art. 117 Abs. 3 StPO übertragen. Nach dieser Bestimmung haben die nahen Angehörigen eines Opfers Verfahrensrechte, wenn sie Zivilansprüche stellen. In diesem Fall sind sie ohne Weiteres legitimiert, Nichtanhandnahme- oder Einstellungsverfügungen mit strafprozessualer Beschwerde anzufechten. [...] Unter dem Blickwinkel des Rechtsgleichheitsgebots (Art. 8 BV6) ist daher nicht einzusehen, weshalb die nahen Angehörigen eines Opfers die Nichtanhandnahme oder Einstellung eines Strafverfahrens bei einer zur Anzeige gebrachten Straftat in einem privaten Spital anfechten können, der kantonale Rechtsmittelweg gegen die Nichtanhandnahme- oder Einstellungsverfügung ihnen dagegen versagt bleibt, wenn sich die mutmassliche Straftat in einem öffentlichen Spital ereignete. Für die betroffenen Angehörigen ist diese Unterscheidung schwer nachvollziehbar [...]. Es liegt darin ein Element der Willkür [...].

11 1.5 Bedeutsam ist in diesem Zusammenhang auch das konventions- und verfassungsrechtlich garantierte Recht auf Leben (Art. 2 Ziff. 1 EMRK7) [...]. Unter Abstützung auf diese Garantien bejahte das Bundesgericht in Fällen staatlicher Gewaltausübung ein rechtlich geschütztes Interesse der Betroffenen an der Auf-

$6 \overline{\text { Bundesverfassung der Schweizerischen Eidge- }}$ nossenschaft vom 18. April 1999 (BV; SR 101).

7 Konvention zum Schutze der Menschenrechte und Grundfreiheiten vom 4. November 1950 (EMRK; SR 0.101). 
hebung eines Entscheids, mit dem die Eröffnung einer Strafuntersuchung gegen einen Beamten abgelehnt oder die Untersuchung eingestellt wurde (BGE 139 IV 121 E. 4.2 [...]). Die Legitimation der geschädigten Person zur Beschwerde in Strafsachen im Sinn von Art. 81 Abs. 1 lit. b BGG wird in diesen Fällen direkt aus den konventions- und verfassungsrechtlichen Garantien abgeleitet [...].

Als weiteren Schritt anerkannte das Bundesgericht den Anspruch auf eine wirksame und vertiefte Untersuchung auch bei fahrlässig begangenen Tötungsdelikten (BGE 135 I 113 E. 2.1) [...].

Der Anspruch auf eine wirksame und vertiefte amtliche Untersuchung (Art. 2 Ziff. 1 EMRK, Art. 10 Abs. 1 BV) legt ebenfalls nahe, den Angehörigen eines Tötungsopfers Parteistellung und somit die Befugnis zur StPO-Beschwerde einzuräumen, wenn sie öffentlich-rechtliche Haftungsansprüche geltend machen [...].

14 1.6 Nach dem Gesagten ist die Legitimation der Beschwerdeführerin als nahe Angehörige des Opfers zur Erhebung einer strafprozessualen Beschwerde gegen die Einstellungsverfügung [...] zu bejahen. [...] Auf die Beschwerde ist einzutreten.

\section{Anmerkungen}

15

«Opfer zweiter Klasse», so bezeichnete Moritz Oehen in dieser Zeitschrift bereits vor mehr als drei Jahren die Opfer staatlicher Gewalt. ${ }^{8}$ Er legte damals dar, dass die strafprozessualen Opferrechte stark

8 Moritz Oehen, Opfer zweiter Klasse: Opfer staatlicher Gewalt und die Beschwerde in Strafsachen, in: sui-generis 2015, S. $34 \mathrm{ff}$. divergieren, je nachdem, ob ein Fussgänger von einem Privatfahrzeug oder von einem Polizeiauto angefahren wird. Übertragen auf den vorliegenden Kontext: Stirbt ein Patient unter dem Messer eines unsorgfältigen Chirurgen, dann macht es strafprozessual einen Unterschied, ob dieser Chirurg seine Klinge im Dienste einer Privatklinik oder eines öffentlichen Spitals führte. Während Angehörige im ersten Fall durch alle Rechtsmittelinstanzen hindurch verlangen können, dass gegen den Arzt der Privatklinik eine Strafuntersuchung geführt wird, ist ihnen dies bei Ärzten von öffentlichen Spitälern verwehrt.

16 Dies ist die Quintessenz einer bundesgerichtlichen Rechtsprechung, welche in der Lehre schon seit Jahren kritisiert wird. ${ }^{9}$ Wer Opfer von staatlicher Polizei-

$9 \overline{\text { Omar Abo Youssef, Die Legitimation des Geschä- }}$ digten zur Beschwerde in Strafsachen - Zugleich Bemerkungen zu BGE 136 IV 29, BGE 136 IV 41 und BGer, Urteil v. 15.4.2010, 6B_127/2010, in: forumpoenale 5/2010, S. 313 ff.; Heinz Aemisegger, Zur Umsetzung der Schweizerischen Strafprozessordnung, in: Angela Cavallo/Eliane Hiestand/Felix Blocher/Irene Arnold/Beatrice Käser/Milena Caspar/Ingo Civic, Liber Amicorum für Andreas Donatsch, Zürich 2012, S. 251 ff., 260 f.; Viktor Lieber, Anmerkung zu Bundesgericht, I. öffentlich-rechtliche Abteilung, Entscheid vom 12.10.2012 i.S. X. c. Obergericht des Kantons Zürich (1B_355/2012), Praxis 102 (2013), Nr. 1; Giusep Nay, Recht haben und Recht bekommen vor Bundesgericht, in: Marcel Alexander Niggli/José Hurtado Pozo/Nicolas Queloz (Hrsg.), Festschrift für Franz Riklin, Zürich 2007, S. 453 ff., 460 ff.; Etienne Vogel, Ungleiche Verkehrsopfer, in: NZZ v. 26.5.2010, Nr. 118, 18; Stefan Keller/Hans Wiprächtiger, Beschwerde in Strafsachen, in: Handbücher für die Anwaltspraxis, Prozessieren vor Bundesgericht, 4. Aufl., Basel 2014, § 3 N 3.46 ff.; Oehen (Fn. 8), S. 35 ff.; Charlotte Schoder, Geschädigte, Opfer und Angehörige mit Staatshaftungsansprüchen. Gelegenheit zur Klärung und Verbesserung ihrer strafprozessualen Stellung im Zuge der Revision von BGG und StPO, in: Jusletter 17. Dezember 2018; sowie meine Kritik in: Die Opfer staatlicher Gewalt vor Bundesgericht, in: Marianne Heer/Stefan Heim- 
gewalt oder amtsärztlicher Unsorgfalt wurde, hat Pech gehabt. Nimmt die Staatsanwaltschaft nicht von sich aus eine Untersuchung auf oder stellt sie eine solche ein, können das Opfer und seine Angehörigen letztlich keine Untersuchung erzwingen. Die Türen zum Bundesgericht bleiben ihnen in aller Regel verschlossen. ${ }^{10}$ Begründet wird dies damit, dass Art. 81 Abs. 1 lit. b Ziff. 5 BGG die Privatklägerschaft nur dann zur Beschwerde in Strafsachen zulässt, wenn «der angefochtene Entscheid sich auf die Beurteilung ihrer Zivilansprüche auswirken kann.» An einer solchen Auswirkung auf zivilrechtliche Haftungsforderungen fehle es, wenn die Betroffenen nur nach öffentlich-rechtlichen Haftungsvorschriften entschädigt werden, wie dies bei der Schädigung durch staatliche Akteure oftmals der Fall ist. ${ }^{11}$

Gegen diese Praxis wird im Wesentlichen eingewendet, dass sie von einem zu en-

gartner/Marcel Alexander Niggli/Marc Thommen (Hrsg), Toujours agité - jamais abattu, Festschrift für Hans Wiprächtiger, Basel 2011, S. 87 ff., 89 ff. und in: Basler Kommentar Bundesgerichtsgesetz, Marcel Alexander Niggli/Peter Uebersax/Hans Wiprächtiger/Lorenz Kneubühler (Hrsg.), 3. Aufl., Basel 2018 (fortan zit.: BSK BGG3-Bearbeiter/in), Art. $81 \mathrm{~N} 39 \mathrm{ff}$.

$10 \mathrm{Zu}$ den Ausnahmen Oehen (Fn. 8), S. $37 \mathrm{ff}$.

11 Vgl. Urteil des Bundesgerichts 6B_1028/2016 vom 10. Februar 2017 E. 1.2 m.H.a. die noch unter altem Verfahrensrecht ergangenen BGE 131 I 455 E. 1.2.4 und BGE 128 IV 188 E. 2.2: «1.2. Als Zivilansprüche im Sinne von Art. 81 Abs. 1 lit. b Ziff. 5 BGG gelten solche, die ihren Grund im Zivilrecht haben und deshalb ordentlicherweise vor dem Zivilgericht durchgesetzt werden müssen. In erster Linie handelt es sich um Ansprüche auf Schadenersatz und Genugtuung nach Art. $41 \mathrm{ff}$. OR. Nicht in diese Kategorie gehören Ansprüche, welche sich aus öffentlichem Recht ergeben. Öffentlich-rechtliche Ansprüche, auch solche aus Staatshaftungsrecht, können nicht adhäsionsweise im Strafprozess geltend gemacht werden und zählen nicht zu den Zivilansprüchen im Sinne von Art. 81 Abs. 1 lit. b Ziff. 5 BGG [...]». gen Verständnis der Haftungsforderungen ausgeht. Nicht nur Zivil-, sondern auch Staatshaftungsgerichte werden de facto durch strafrechtliche Entscheide beeinflusst. ${ }^{12}$ Deshalb haben gerade auch die Opfer staatlicher Gewalt oder Unsorgfalt ein rechtliches Interesse daran, Nichtanhandnahmen, Verfahrenseinstellungen und Freisprüche anfechten zu können. ${ }^{13}$ Verwehrt man ihnen dieses Recht, macht man sie zu Opfern zweiter Klasse resp. behandelt sie ohne sachlichen Grund ungleich. ${ }^{14}$

18 Das Obergericht des Kantons Zürich hat nun entschieden, dass diese strikte Eintretenspraxis zur Beschwerde in Strafsachen nicht gilt für StPO-Beschwerden von Opferangehörigen gegen Einstellungsentscheide der Staatsanwaltschaft.

19 Dem Entscheid ist im Ergebnis klar zuzustimmen. Opferangehörige müssen sich gegen Verfahrenseinstellungen wehren können unabhängig davon, ob die mutmasslichen Schädiger zivil- oder öffentlich-rechtlichem Haftungsrecht unterstehen. In der Begründung ist der Entscheid zu sehr auf die Rechte der Opferangehörigen fixiert (1.) und blendet damit die wichtige Vorfrage aus, ob sich die Betroffene überhaupt als Privatklägerin konstituieren konnte (2.). Erst wenn man dies nämlich mit der Rechtsprechung und wohl noch herrschenden Lehre verneint, stellt sich die Frage, ob man die Interpretation der Zivilansprüche überdenken (3.) oder allenfalls die Legitimation zur Beschwerde erweitern muss (4.).

12 Oehen (Fn. 8), S. 42.

13 Schoder (Fn.9), Rz. 12

14 Vogel (Fn. 9), 18. 


\section{Rechte von Opferangehörigen}

20 Ausgangspunkt der obergerichtlichen Erwägungen ist einerseits Art. 117 Abs. 3 StPO, wonach Angehörigen des Opfers die gleichen Rechte wie dem Opfer zustehen, wenn sie Zivilansprüche geltend machen. Andererseits wird auf Art. 122 Abs. 2 StPO abgestellt, wonach die Angehörigen von Opfern ihre Zivilklage dann adhäsionsweise in das Strafverfahren einbringen können, wenn sie eigene Zivilansprüche haben (in casu Versorgerschaden und Genugtuung). Dies führe zur Frage, ob den Opferangehörigen, die anstelle einer zivilrechtlichen eine öffentlich-rechtliche Forderung geltend machen, die Berechtigung zur Anfechtung einer Nichtanhandnahme- oder Einstellungsverfügung fehlt.

Von diesem Punkt aus entwickelt das Obergericht seine berechtigte Kritik an der bundesgerichtlichen Interpretation der Zivilansprüche, ohne jedoch auf die Vorfrage einzugehen, ob sich Opferangehörige überhaupt als Privatklägerinnen konstituieren können.

\section{Konstituierung als Privatklägerin}

Überzeugender wäre es gewesen, wenn das Obergericht zuerst zur Frage Stellung genommen hätte, ob die Tochter des Verstorbenen sich gültig als Privatklägerin konstituiert hatte. Als Privatklägerschaft gilt die geschädigte Person, die ausdrücklich erklärt, sich am Strafverfahren als Straf- oder Zivilklägerin zu beteiligen (Art. 118 Abs. 1 StPO). Geschädigte Person ist vorliegend der wegen Leberkrebs operierte Patient (Art. 115 Abs. 1 StPO). Weil er im Gefolge der Operation verstarb, bestand der Verdacht, dass er Opfer einer Straftat wurde (Art. 116 Abs. 1 StPO). Sei- ne Tochter ist Angehörige im Sinne von Art. 116 Abs. 2 StPO. Sie hat deshalb das Recht, sich als Privatklägerin im Strafund Zivilpunkt zu konstituieren, ${ }^{15}$ wobei das «und» hier so zu verstehen ist, dass die Angehörige Strafklage nur erheben kann, wenn sie zugleich eigene Zivilforderungen geltend macht. ${ }^{16}$ Das ergibt sich unmittelbar aus dem Gesetzeswortlaut von Art. 117 Abs. 3 StPO: «Machen die Angehörigen des Opfers Zivilansprüche geltend, so stehen ihnen die gleichen Rechte zu wie dem Opfer.» Es ist deshalb zu prüfen, ob die Tochter sich gültig als Straf- und Zivilklägerin konstituiert hatte:

23 Für die Konstituierung als Strafklägerin reicht die blosse Strafanzeige bekanntlich nicht, ${ }^{17}$ vielmehr muss im Sinne von Art. 119 Abs. 2 lit. a StPO der Wille zum Ausdruck gebracht worden sein, dass die verantwortliche Person verfolgt und bestraft werde. Vorliegend ist wohl davon auszugehen, dass die Tochter nicht nur den Umstand, dass (mutmasslich) eine Straftat begangen wurde zur Anzeige bringen wollte, sondern sie auch die Verfolgung und Bestrafung derjenigen Per-

15 BGE 139 IV 89 E. 2.2.

16 Implizit BGE 139 IV 89 E. 2.2. i.f. («Autrement dit, le proche de la victime ne peut se constituer partie plaignante que s'il fait valoir des prétentions civiles propres dans la procédure pénale»), explizit Goran Mazzucchelli/Mario Postizzi, in: Marcel Alexander Niggli/Marianne Heer/Hans Wiprächtiger (Hrsg.), Basler Kommentar Schweizerische Strafprozessordnung / Jugendstrafprozessordnung (StPO/JStPO), 2. Aufl., Basel 2014 (fortan zit.: BSK StPO²-Bearbeiter/in), Art. 115 $\mathrm{N} 11$ («...vorausgesetzt, sie machen eigene privatrechtliche Ansprüche (etwa aus Art. 45 Abs. 3, Art. 47 OR) adhäsionsweise geltend... Die Strafklage ohne gleichzeitige Zivilklage für eigene Ansprüche ist ihnen verwehrt»).

17 BSK StPO 2-Mazzucchelli/Postizzi, Art. 118 N 7 («Nicht als Konstituierung gilt - zumindest bei Offizialdelikten - die Strafanzeige, mit welcher die Behörde über das Bestehen eines bestimmten Sachverhalts informiert wird»). 
sonen im Blick hatte, welche ihres Erachtens für den Tod ihres Vaters verantwortlich waren. Es ist also davon auszugehen, dass sie sich als Strafklägerin konstituieren wollte. Dies kann sie aber wie gesehen nur, wenn sie sich zugleich gültig als Zivilklägerin konstituiert hat.

24 Für die Zivilklage ist vorausgesetzt, dass adhäsionsweise privatrechtliche Ansprüche geltend gemacht werden, die aus der Straftat abgeleitet werden (Art. 119 Abs. 2 lit. b StPO). ${ }^{18}$ Keine adhäsionsfähigen Forderungen im Sinne dieser Bestimmung sind nach ständiger Rechtsprechung und wohl noch h.L. öffentlichrechtliche Ansprüche aus Staatshaftung. ${ }^{19}$ Folgt man dieser Interpretation, dann hätte sich die Tochter im vorliegenden Fall nicht als Zivilklägerin konstituieren können. Denn wie das Obergericht zutreffend ausgeführt hat, richten sich allfällige Forderungen gegen das involvierte Medizinalpersonal des USZ nach dem kantonalen öffentlich-rechtlichen Haftungsgesetz. Weil Art. 117 Abs. 3 StPO die Zivil- und Strafklägerposition zudem untrennbar miteinander verknüpft, bedeutet dies für den vorliegenden Fall, dass sich die Tochter gar nicht als Privatklägerin hätte konstituieren können. Ohne den Status als Privatklägerin war sie nicht Verfahrenspartei (Art. 104 Abs. 1 lit.b StPO). Weil nach Art. 322 Abs. 2 StPO nur die Parteien die Einstellungsverfügung

$18 \overline{\text { Eingehend Christina Galeazzi, der Zivilkläger im }}$ Strafbefehls- und abgekürzten Verfahren, Diss. Zürich 2016, S. 54 ff.

19 BGE 141 IV 380 E. 2.3.1 m.H.a. BGE 131 I 455 E. 1.2.4; BGE 128 IV 188 E. 2.2 und Urteil des Bundesgerichts 6B_1168/2014 vom 13. Februar 2015 E. 1; BSK StPO2-Mazzucchelli/Postizzi, Art. 119 N 10; Bettina Alexandra Tanner, Das Teilnahmerecht der Privatklägerschaft nach Art. 147 StPO und seine Grenzen, Diss. Zürich 2018, S. 67. anfechten können, war sie nicht zur Beschwerde gegen die Verfahrenseinstellung legitimiert.

25 Aus dieser Situation gibt es nun zwei Auswege: Entweder man hinterfragt die dem Ausschluss von der Zivilklage zugrundliegende Interpretation der Zivilansprüche (3.) oder man erweitert die Legitimation zur Beschwerde gegen Einstellungsentscheide auf weitere Betroffene (4.).

\section{Interpretation der Zivilansprüche}

26 Mit Blick auf den zu beurteilenden Fall geht es in erster Linie um die Frage, ob Opferangehörige, die keine Zivilforderungen i.e.S. haben, sich als Privatklägerinnen konstituieren können. Darüber hinaus strahlen die folgenden Überlegungen aber auch auf alle durch staatliches Handeln geschädigten Personen aus. Betroffen sind also auch direkte Opfer staatlicher Gewalt und Unsorgfalt sowie Geschädigte staatlicher Vermögensdelikte. Sie alle haben nach der herkömmlichen Diktion nämlich keine Zivilansprüche i.e.S.

27 Es rechtfertigt sich deshalb, die Regelungen des Bundesgerichtsgesetzes und der Strafprozessordnung anzuschauen: Nach Art. 81 Abs. 1 lit. b Ziff. 5 BGG ist die Privatklägerschaft zur Beschwerde in Strafsachen an das Bundesgericht legitimiert, wenn sich der angefochtene Entscheid auf die Beurteilung ihrer Zivilansprüche auswirken kann. Nach Art. 119 Abs. 2 lit. b StPO kann die Geschädigte in der Zivilklage adhäsionsweise privatrechtliche Ansprüche geltend machen, die aus der Straftat abgeleitet werden. Die Begriffe werden nach herkömmlicher Leseart synonym interpretiert als Forderungen, 
die ihren Grund im Zivilrecht haben und deshalb vor dem Zivilgericht durchgesetzt werden müssen. ${ }^{20}$ «Forderungen öffentlich-rechtlicher Natur werden vom Strafrichter nicht beurteilt.»21

Gegen eine solche Interpretation ist aus grammatikalischer Sicht nichts einzuwenden. In der Tat verstehen Juristen unter den Begriffen «Zivilansprüche» resp. «privatrechtliche Ansprüche» solche, die ihren Ursprung im Zivilrecht haben. Damit ist aber noch nicht gesagt, dass der Gesetzgeber tatsächlich nur Ansprüche aus dem Zivilrecht meinte, als er die Regelungen in Art. 81 BGG und Art. 119 StPO aufstellte.

29 Ein Blick in die Gesetzgebungsgeschichte zeigt, dass zwar hart darum gerungen wurde, ob die sog. einfachen Geschädigten ${ }^{22}$ zur Beschwerde in Strafsachen zuzulassen seien, ${ }^{23}$ die bundesgerichtliche Differenzierung zwischen zivil- und öffentlich-rechtlichen Haftungsforderungen war jedoch zu keinem Zeitpunkt ein Thema, weder bei den Beratungen zu Art. 81 BGG noch bei denjenigen zu Art. 119 StPO. ${ }^{24}$ Die Lektüre der Materia-

20 BGE 141 IV 1 E. 1.1. («Constituent de telles prétentions celles qui sont fondées sur le droit civil et doivent en conséquence être déduites ordinairement devant les tribunaux civils»).

21 Tanner (Fn. 19), S. 67.

22 Damit sind unmittelbar deliktsbetroffene Personen gemeint, die keinen Opferstatus haben, also nicht in ihrer körperlichen, sexuellen oder psychischen Integrität beeinträchtigt wurden. Beispiele sind etwa bestohlene oder betrugsgeschädigte Personen.

23 Eingehend zur Gesetzgebungsgeschichte BSK BGG3-Thommen, Art. $81 \mathrm{~N} 24 \mathrm{ff}$.

24 S.a. Parlamentarische Initiative Nr. 12.492 von Mauro Poggia vom 11. Dezember 2012, Zulassung zum Bundesgericht. Beseitigung der ungerechtfertigten Ungleichbehandlung von Opfern («Eine solche Ungleichbehandlung ist ungerechtfertigt und wurde vom Parlament sicherlich nicht beabsichtigt»). lien legt vielmehr den Schluss nahe, dass dem Gesetzgeber nicht bewusst war, dass mit den Betroffenen von staatlicher Gewalt und hoheitlicher Unsorgfalt eine ganze Opferkategorie schon damals von der Beschwerde ausgeschlossen war und es auch nach neuem Recht bleiben würde. ${ }^{25}$

30 Systematisch sind die Bestimmungen zur Privatklägerschaft von Art. $118 \mathrm{ff}$. StPO eingebettet in das 3. Kapitel zu den Geschädigten- und Opferrechten (Art. 115 ff. StPO), in welchem gleich zu Beginn klargestellt wird, dass als geschädigte Person gilt, wer durch die Straftat in seinen Rechten unmittelbar verletzt worden ist. Für die Qualifikation als Opfer wird sodann auf die Intensität dieser unmittelbaren Verletzung abgestellt: Als Opfer gilt die geschädigte Person, die durch die Straftat in ihrer körperlichen, sexuellen

$25 \overline{\text { Vgl. etwa Eidgenössisches Justiz- und Polizeide- }}$ partement, Bundesamt für Justiz, Begleitbericht zum Vorentwurf für eine Schweizerische Strafprozessordnung, Bern, Juni 2001, S. 82 ff.; Botschaft zur Vereinheitlichung des Strafprozessrechts vom 21. Dezember 2005 (BBl 2005 1085), 1171 ff.; in der Botschaft zum Bundesgesetz über die Organisation der Strafbehörden des Bundes (Strafbehördenorganisationsgesetz, StBOG) vom 10. September 2008 (BBl 2008 8125), 8182 f., wo es darum ging, die durch die Strafprozessordnung eingeführte Erweiterung der Beschwerdelegitimation der als Privatklägerschaft konstituierten einfachen Geschädigten wieder rückgängig zu machen, hat der Bundesrat nicht einmal erwähnt, dass in den hier diskutierten Fällen sogar die Opfer von der Beschwerde ausgeschlossen sind. Das Gleiche gilt auch für die Parlamentsdebatte, vgl. Sitzung des Ständerats vom 1. März 2010, Geschäftsnummer 08.066 (AB $2010 \mathrm{~S} 3$ ), 8 f. und Sitzung des Nationalrats vom 3. März 2010, Geschäftsnummer 08.066 (AB 2010 N 117), 122 ff.; diese Einschätzung, dass dem Bundesgesetzgeber die bundesgerichtliche Differenzierung zwischen Opfern privater und öffentlicher Akteure nicht klar vor Augen steht, trifft auch auf die jüngste BGG-Revision zu: Botschaft zur Änderung des Bundesgerichtsgesetzes (BGG) vom 15. Juni 2018 (BBl 2018 4605), $4615 \mathrm{ff}$. 
oder psychischen Integrität unmittelbar beeinträchtigt worden ist (Art. 116 Abs. 1 StPO). Weder für die Geschädigten- noch für die Opferstellung wird somit danach differenziert, in welcher Qualifikation der Schädiger gehandelt hat. Ob der Schädiger ein Privater oder eine Person mit Beamten- oder Behördenstatus war, spielt keine Rolle. Entscheidend ist lediglich die direkte Schädigung. Auch die systematische Sichtweise spricht somit dagegen, von staatlichen Akteuren geschädigten Personen weniger Rechte einzuräumen.

Das gleiche Resultat legt auch eine verfassungskonforme Auslegung nahe. Sowohl die Verfassungsbestimmung zur Opferhilfe als auch das Opferhilfegesetz wurden geschaffen, um Zivilforderungen von Opfern direkt im Strafverfahren beurteilen zu können. ${ }^{26}$ Den Opfern sollten weitere Verfahren erspart bleiben. ${ }^{27} \mathrm{Ge}-$ nau diesem Ziel widerspricht die Praxis, Opfer staatlicher Gewalt- oder Fahrlässigkeitsdelikte systematisch in ein separates Staatshaftungsverfahren zu verweisen. ${ }^{28}$

$26 \overline{\text { Botschaft zu einem Bundesgesetz über die Hilfe }}$ an Opfer von Straftaten (Opferhilfegesetz, OHG) und zu einem Bundesbeschluss über das Europäische Übereinkommen über die Entschädigung für Opfer von Gewalttaten vom 25. April 1990 (BBl 1990 II 961), 962; umfassend zur Entwicklung der Opferrechte Felix Bommer, Offensive Verletztenrechte im Strafprozess, Habil. Bern 2006, S. $1 \mathrm{ff}$.

27 Siehe etwa Botschaft zur Volksinitiative «zur Entschädigung der Opfer von Gewaltverbrechen» vom 6. Juli 1983 (BBl 1983 III 869), 882.

28 Vgl. Parlamentarische Initiative Nr. 12.463 von Mauro Poggia vom 11.9.2012, Privatklägerschaft im Strafprozess. Schliessung einer Gesetzeslücke («Indem es also zwei Kategorien von Angehörigen gibt, entsteht eine Ungleichheit, die sich keineswegs rechtfertigen lässt und die sogar gegen die Idee des Strafrechts verstösst, das zum Ziel hat, den Opfern ein einfaches und effizientes Mittel zur Verfügung zu stellen, um die Personen, die eine strafbare Handlung gegen Leib und Leben
32 Schliesslich sprechen teleologische Erwägungen für eine weite Beschwerdelegitimation der Privatklägerschaft. Hierzu muss man sich die Frage stellen, weshalb die Privatkläger im Normalfall, d.h. bei Schädigungen durch private, nach Zivilrecht haftende Täter, zur Beschwerde gegen Freisprüche, Einstellungen und Nichtanhandnahmen legitimiert sind. Hier ist die Antwort, dass trotz Art. 53 OR, wonach ein strafrechtlicher Freispruch für das Zivilgericht nicht bindend ist, selbst das Bundesgericht davon ausgeht, dass der Zivilrichter "faktisch an den Strafentscheid gebunden ist».29 Kein Zivilgericht wird ohne triftigen Grund einen gerichtlichen Freispruch ignorieren. Noch weniger wird es eine staatsanwaltschaftliche Nichtanhandnahme oder Einstellung übergehen, weil hier die Hürden noch höher sind. Während das Strafgericht im Zweifel freisprechen muss, hat die Staatsanwaltschaft in dubio pro duriore Anklage zu erheben. Sie darf also nur einstellen, wenn mit Sicherheit kein Tatbestand erfüllt ist. 30

33 Das Anfechtungsrecht hat also den Sinn, die Entschädigungschancen der Privatklägerin zu wahren. Sie muss verhindern können, dass durch den Strafentscheid Fakten geschaffen werden, welche ihren Entschädigungsanspruch schmälern. Wie das Obergericht zu Recht festgehalten hat, besteht die Gefahr einer solchen Beeinflussung durch den Einstellungsentscheid unabhängig davon, ob sich der

begangen haben, auch rechtlich belangen zu können»); BSK BGG3-Thommen, Art. 81 N 39 («Eine Verweisung der Opferansprüche in ein Staatshaftungsverfahren widerspricht der ratio legis nur schon deshalb, weil das Opfer ein separates zweites Verfahren anstrengen muss»).

29 BGE 120 IV 44 E. 6; BGE 120 Ia 101 E. 2e; BSK BGG3-Thommen Art. $81 \mathrm{~N} 21 \mathrm{c}$ m.w.H.

3о ZHK StPO'-Landshut/Bosshard, Art. 319 N 16. 
Entschädigungsanspruch nach zivilem oder öffentlichem Haftungsrecht beurteilt. Das zeigt sich besonders deutlich im Kanton Zürich, wo Staatshaftungsansprüche durch die gleichen Zivilgerichte beurteilt werden wie die Haftungsansprüche aus dem Privatrecht. ${ }^{31}$

34 Eine umfassende Interpretation der einschlägigen Bestimmungen bestätigt somit die obergerichtliche Schlussfolgerung, dass die Begriffe «Zivilansprüche» (Art. 81 Abs. 1 lit. b Ziff. 5 BGG) und «privatrechtliche Ansprüche» (Art. 119 Abs. 2 lit. b StPO) auch auf Staatshaftungsansprüche auszudehnen sind. Im Ergebnis bedeutet dies, dass die Tochter des Verstorbenen sich als Privatklägerin konstituieren und in dieser Eigenschaft die Verfahrenseinstellung anfechten konnte (Art. 322 Abs. 2 und Art. 382 Abs. 1 StPO). Die Bedeutung dieses Ergebnisses geht jedoch weit über die Opferangehörigen hinaus. Wenn man solche Forderungen unabhängig von ihrem Rechtsgrund im Zivil- oder öffentlichen Recht als Haftungsforderungen interpretiert, dann können sich alle Personen, die von staatlichen Akteuren geschädigt wurden, als Privatklägerinnen konstituieren und als solche Beschwerde gegen Einstellungsentscheide führen.

\section{Legitimation zur Beschwerde}

35 Die Auslegung der Begriffe «Zivilansprüche» und "privatrechtliche Ansprüche» hat gezeigt, dass Geschädigte, Opfer und deren Angehörige sich auch als Privatklägerinnen sollen konstituieren können, wenn ihnen alleine öffentlich-rechtliche Haftungsansprüche offenstehen. Obwohl

${ }_{31} \S 19$ f. Haftungsgesetz. das Bundesgericht eingeräumt hat, dass «nachvollziehbare Gründe» für eine solche Interpretation sprechen, fühlt es sich de lege lata an den «klaren Wortlaut» gebunden. ${ }^{22}$ Zumindest für die StPO-Beschwerde besteht hier auf den ersten Blick noch ein Ausweg: Unter der Überschrift, Legitimation der übrigen Parteien' bestimmt Art. 382 Abs. 1 StPO: «Jede Partei, die ein rechtlich geschütztes Interesse an der Aufhebung oder Änderung eines Entscheides hat, kann ein Rechtsmittel ergreifen». Obwohl das Gesetz von «Partei» spricht, besteht Einigkeit, dass der Begriff umfassend zu verstehen ist. Nicht nur Beschuldigte, Staatsanwaltschaften oder Privatklägerinnen sollen zum Rechtsmittel zugelassen sein, sondern jedermann, der beschwert ist. 33

36 Für den vorliegenden Fall bedeutete dies, dass selbst wenn man die Tochter des Verstorbenen nicht als Privatklägerin einstufen wollte, sie als «durch Verfahrenshandlungen beschwerte Dritte» (Art. 105 Abs. 1 lit. f StPO) zur Beschwerde zuzulassen ist, weil sich der Einstellungsentscheid auf ihre Staatshaftungsansprüche auswirkt. Über diesen konkreten Fall hinaus, könnten alle von staatlichen Akteuren unmittelbar geschädigten Perso-

32 Urteil des Bundesgerichts 6B_945/2013 vom 23. Mai 2014 E. 3.3.1.

33 Botschaft (Fn. 25; BBl 2005 1085), 1308 («Absatz 1 enthält keine abschliessende Liste der zur Beschwerde legitimierten Parteien. Der Parteibegriff ist im Sinn der Artikel 102 und 103 zu verstehen. Demnach wird nebst der beschuldigten Person und der Privatklägerschaft auch den anderen am Verfahren beteiligten Personen die Beschwerdelegitimation zuerkannt, sofern sie sich am erstinstanzlichen Verfahren beteiligt haben und ein rechtlich geschütztes Interesse geltend machen können»); BGE 139 IV 78 E. 3.1 («La notion de partie visée à l'art. 382 CPP doit être comprise au sens des art. 104 et 105 CPP»); s. auch Art. 105 Abs. 2 StPO. 
nen als «übrige Parteien» zur Beschwerde gegen Einstellungen und Nichtanhandnahmen zugelassen werden. Das Gleiche gilt selbstredend auch für die Anfechtung gerichtlicher Freisprüche.

Bei näherem Hinsehen erweist sich diese Lösung jedoch aus drei Gründen als weniger überzeugend: Erstens zeigt allein schon die Bezeichnung als «übrige» Parteien, dass die Betroffenen immer noch Opfer zweiter Klasse sind. Zweitens wären diese Personen dann immer noch von der Beschwerde an das Bundesgericht ausgeschlossen, weil hier nicht Art. 382 StPO, sondern Art. 81 BGG anwendbar ist. Wie das Obergericht $\mathrm{zu}$ Recht betont, wird damit das Rechtsgleichheitsgebot (Art. 8 BV) verletzt.34 Drittens schliesslich geht die oben vorgeschlagene Lösung weit über die Beschwerdelegitimation hinaus. Indem die Betroffenen als Privatkläger zugelassen werden, können sie nicht nur Rechtsmittel erheben, sondern von allem Anfang an als gleichberechtigte Parteien an der Untersuchung teilnehmen und bei der Erstellung des Sachverhalts mitwirken.

\section{Fazit}

38 Zusammenfassend wurde gezeigt, dass der Beschluss des Obergerichts des Kantons Zürich vom 24. April 2018 wichtig

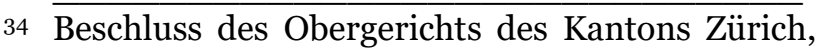
III. Strafkammer, UE170255 vom 24. April 2018 E. 1.4: «Unter dem Blickwinkel des Rechtsgleichheitsgebots (Art. $8 \mathrm{BV}$ ) ist daher nicht einzusehen, weshalb die nahen Angehörigen eines Opfers die Nichtanhandnahme oder Einstellung eines Strafverfahrens bei einer zur Anzeige gebrachten Straftat in einem privaten Spital anfechten können, der kantonale Rechtsmittelweg gegen die Nichtanhandnahme- oder Einstellungsverfügung ihnen dagegen versagt bleibt, wenn sich die mutmassliche Straftat in einem öffentlichen Spital ereignete». und richtig war. Für Opferangehörige ist es schlicht nicht nachvollziehbar, weshalb sie eine Einstellung anfechten können, wenn eine nahestehende Person durch einen privatrechtlich agierenden Arzt getötet wurde, ihnen hingegen die Hände gebunden sind, wenn sich der gleiche Vorfall in einem öffentlichen Spital ereignet hat. $\mathrm{Zu}$ diesem Resultat gelangte die Rechtsprechung in einer am Wortlaut klebenden Interpretation der «Zivilansprüche». Es wurde gezeigt, dass alle übrigen Auslegungselemente dafür sprechen, den Begriff der Zivilansprüche in einem weiteren Sinne als Haftungsansprüche zu interpretieren. Im Ergebnis bedeutete dies, dass Personen, die von staatlichen Akteuren unmittelbar geschädigt werden, sich als Privatkläger konstituieren und als solche durch alle Instanzen Rechtsmittel gegen Nichtanhandnahmen, Einstellungen und Freisprüche ergreifen können. Das Gleiche gilt auch für die Opferangehörigen nach Art. 117 Abs. 3 StPO.

39 Dass die von der Rechtsprechung getroffene Differenzierung unsachgemäss ist, wurde unterdessen auch in Bern erkannt. Insbesondere der damalige Nationalrat Mauro Poggia hat sich bereits 2012 für dieses Anliegen stark gemacht. In seiner Initiative 12.463 vom 11. September 2012 wurde verlangt, Art. 118 StPO dahingehend zu ergänzen, dass Angehörige verstorbener Opfer sich als Privatklägerschaft konstituieren können, selbst wenn sie gegen den Täter keine Zivilklage erheben können. Seine Initiative 12.492 vom 11. Dezember 2012 verlangte, das Beschwerderecht im bundesgerichtlichen Verfahren zu erweitern auf Privatkläger mit öffentlich-rechtlichen Forde- 
rungen. Beiden parlamentarischen Initiativen wurde Folge gegeben. 35

$40 \mathrm{Ob}$ das erste Anliegen, alle Opfer und Geschädigten gleich zu behandeln, in der revidierten Strafprozessordnung aufgenommen wird, ist derzeit noch offen. Der Entwurf soll Mitte 2019 vorgelegt werden. ${ }^{6}$ Die zweite parlamentarische Initiative wurde im Entwurf zum revidierten Bundesgerichtsgesetz leider nicht umgesetzt.37 Ganz im Gegenteil wird im Entwurf - man muss sagen: schon wieder versucht, das Rad der Zeit zurückzudrehen und die Legitimation der Privatklägerschaft generell einzuschränken. Es bleibt zu hoffen, dass sich das Parlament davon unbeeindruckt zeigen und das Beschwerderecht im Bundesgerichtsgesetz sowie die Parteistellung in der Strafprozessordnung im dargestellten Sinne ausdehnen wird auf Privatkläger, deren Haftungsforderungen betroffen sind. 38

Seit über einem Jahrzehnt schreibt eine wachsende Zahl von AutorInnen gegen die Einteilung von Opfern in zwei Klassen an. ${ }^{39}$ Gutta cavat lapidem non vi sed

35 Parlamentarische Initiative Nr.12.463 (Fn. 28), Parlamentarische Initiative Nr. 12.492 (Fn. 24): «Eine solche Ungleichbehandlung ist ungerechtfertigt und wurde vom Parlament sicherlich nicht beabsichtigt».

36 Mündliche und an dieser Stelle zu verdankende Auskunft vom 21. Januar 2019 von Dr. iur. Peter Goldschmid, Fürsprecher, der beim Bundesamt für Justiz für die Revision der Strafprozessordnung zuständig ist.

37 Botschaft (Fn. 25; BBl 2018 4605) 4615 ff.; zum Entwurf der Änderung des Bundesgerichtsgesetzes (BGG) siehe BBl 20184663 ff.

38 Der Nationalrat hat die Gelegenheit bereits verpasst, für die Beschwerde ans Bundesgericht Gleichheit $\mathrm{zu}$ schaffen. In der Sitzung vom 13. März 2019 hat er Art. 81 Abs. 1 Bst. b Ziff. 5 EBGG diskussionslos angenommen (Amtl. Bull. NR noch nicht verfügbar).

39 Nachweise oben Fn. 9. saepe cadendo.40 Steter Tropfen höhlt bekanntlich jeden Stein. Die Gemäuer des Zürcher Obergerichts hat die Kritik an der Interpretation der Zivilforderungen bereits durchdrungen, nun bleibt als letzter harter Brocken die Festung auf dem Mon Repos zu knacken. Erste Anzeichen für einen möglichen Meinungsumschwung hat das Bundesgericht bereits durchblicken lassen, indem es einräumte, dass «nachvollziehbare Gründe dafür sprechen, den Privatkläger unabhängig der Rechtsnatur seines allfälligen Entschädigungsanspruchs zur Beschwerde in Strafsachen zuzulassen», sich aber letztlich - wer will ihm das übel nehmen? - durch den «klaren Wortlaut» von Art. 81 BGG gebunden sah.41 Nun stellt sich die Frage, wer sich zuerst bewegt, der Bundesgesetzgeber oder das Bundesgericht.
40 Wörtlich: Der Tropfen höhlt den Stein nicht durch Kraft, sondern durch stetiges Fallen (eigene Übersetzung).

41 Urteil des Bundesgerichts 6B_945/2013 vom 23. Mai 2014 E. 3.3.1. 